\title{
Loss of CSMD1 expression is associated with high tumour grade and poor survival in invasive ductal breast carcinoma
}

\author{
Mohamed Kamal · Abeer M. Shaaban · Liqun Zhang • \\ Clare Walker · Sally Gray · Nalin Thakker · \\ Carmel Toomes · Valerie Speirs · Sandra M. Bell
}

Received: 22 July 2009/Accepted: 25 July 2009/Published online: 8 August 2009

(C) Springer Science+Business Media, LLC. 2009

\begin{abstract}
CUB and SUSHI multiple domain protein 1 (CSMD1) is a candidate tumour suppressor gene that maps to chromosome $8 \mathrm{p} 23$, a region deleted in many tumour types including $50 \%$ of breast cancers. CSMD1 has homologies to proteins implicated in carcinogenesis. We aimed to study the expression pattern of the CSMD1 protein and evaluate its prognostic importance in invasive ductal carcinoma (IDC). An anti-CSMD1 antibody was developed and validated. The expression pattern of CSMD1 in normal breast and IDC
\end{abstract}

Electronic supplementary material The online version of this article (doi:10.1007/s10549-009-0500-4) contains supplementary material, which is available to authorized users.

M. Kamal · C. Walker · C. Toomes · S. M. Bell ( $)$

Leeds Institute of Molecular Medicine, University of Leeds,

Wellcome Trust Brenner Building, Level 8, St. James's

University Hospital, Leeds LS9 7TF, UK

e-mail: medsmb@leeds.ac.uk

M. Kamal

Department of Zoology, Faculty of Science, University

of Benha, Benha, Egypt

A. M. Shaaban · C. Walker · S. Gray

St James's Institute of Oncology, St James's University Hospital,

Leeds LS9 7TF, UK

L. Zhang $\cdot$ N. Thakker

Department of Medical Genetics, University of Manchester,

Manchester M13 0JH, UK

V. Speirs

Leeds Institute of Molecular Medicine, University of Leeds,

Wellcome Trust Brenner Building, Level 4, St. James's

University Hospital, Leeds LS9 7TF, UK samples was investigated by immunohistochemistry in 275 patients. Univariate and multivariate Cox regression analyses were performed. In normal breast duct epithelial cells, luminal, membranous and cytoplasmic CSMD1 staining was identified. Reduced expression of CSMD1 was detected in 79/275 (28.7\%) of IDC cases. Low CSMD1 expression was significantly associated with high tumour grade $(P=0.003)$. CSMD1 expression was associated with overall survival $(\mathrm{OS} ; \mathrm{HR}=0.607,95 \% \mathrm{CI}:$ 0.4-0.91, $P=0.018$ ) but not with disease-free survival (DFS; $\mathrm{HR}=0.81,95 \% \mathrm{CI}: 0.46-1.43, P=0.48$ ). Multivariate analysis showed that CSMD1, together with Nottingham Prognostic Index, was considered an independent predictor of OS (HR $=0.607,95 \%$ CI: $0.4-0.91, P=0.018$ ) but not DFS (HR $=0.84,95 \%$ CI: $0.46-1.5, P=0.573$ ). Reduction of CSMD1 expression was significantly associated with high tumour grade and decreased OS. Therefore, our results support the idea that CSMD1 is a tumour suppressor gene and suggest its possible use as a new prognostic biomarker. The membrane expression pattern of CSMD1 suggests that it may be a receptor or co-receptor involved in the process of signal transduction.

Keywords CSMD1 - Breast cancer - Prognosis · Differentiation · Immunohistochemistry

\section{Introduction}

Breast cancer is one of the leading causes of death in women $[1,2]$. It is a complex and heterogeneous disease at the molecular level. Abnormal expression of growth factor receptors, cell-cycle regulatory molecules and cell-adhesion proteins frequently occurs [3]. Investigating the 
expression and functions of these molecules, and their association with patient outcome, is extremely important to aid the understanding of the disease process and assist in clinical decision-making [4].

CUB and SUSHI multiple domains-1 (CSMD1) encodes multiple mRNA transcripts with the largest being $14.3 \mathrm{~Kb}$ long. The gene spans over $2 \mathrm{Mb}$ of genomic DNA and contains 71 exons, which encode a 3,565 amino acid protein consisting of 14 CUB domains and 28 SUSHI domains. CSMD1's function is unknown; however, CUB and SUSHI domains are thought to be involved in receptorligand interactions. The full-length CSMD1 protein is predicted to be a membrane protein with an extracellular region containing the CUB and SUSHI domains, a single transmembrane domain and a short cytoplasmic domain that contains a tyrosine phosphorylation site. From these features, one could predict that CSMD1 is a receptor or coreceptor for unknown ligand(s) and is involved in signal transduction $[5,6]$.

Many lines of evidence indicate CSMDI could be a tumour suppressor gene. Firstly, CSMDI spans chromosomal band 8p23, which is known to exhibit frequent deletions in many cancers, including breast cancer [7-15]. Secondly, loss of CSMD1 has been reported in many cancers. At the DNA level, an array-based comparative genomic hybridization (CGH) study detected the loss of CSMD1 in a large proportion of head and neck squamous cell carcinoma (HNSCC), lung squamous cell carcinoma (SCC) and breast cancers [16]. At the RNA level, quantitative real-time PCR (qPCR) detected the loss of CSMD1 expression in HNSCCs and lung SCCs [16], liver [17], colorectal [18] and prostate cancer [19, 20]. Thirdly, CSMD1 shares homologies with many other proteins playing roles in cancer progression by the virtue of its CUB and SUSHI domains such as SEZ6L and DMBT1. SEZ6L is homozygously deleted in small cell lung cancer cell lines [5, 21, 22], and DMBT1 expression is reduced in malignant brain tumours [23], lung cancer [24] and breast cancer [25]. A variety of developmental proteins have been implicated in carcinogenesis, and CUB and SUSHI domains are also found in many of these, including bone morphogenetic proteins, tolloid, neuropilin and spermadhesins [26-29].

The genomic localisation, expression pattern and protein homology of CSMD1 suggest that it is a tumour suppressor gene. In this study, we investigate its role in breast cancer progression. We have generated an anti-CSMD1 antibody and identified the expression pattern of CSMD1 in 275 IDC patients with a follow-up period of 152 months. Univariate and multivariate Cox regression analyses have been performed to correlate CSMD1 expression with OS and DFS. Loss of CSMD1 expression was associated with high tumour grade and poor survival outcome.

\section{Materials and methods}

Patients

All patients gave informed consent to participate in this study, and ethical approval was obtained from the Local Research Ethics Committee of the Leeds Teaching Hospitals (LTH), NHS Trust, Leeds, UK. Paraffin blocks from two independent cohorts of breast cancer cases were retrieved from the archives of the pathology departments of the LTH, NHS Trust. The first cohort containing 50 cases was designated as the training set, and the second cohort containing 446 cases was used as the validation set. These cases were selected from patients presenting between 1987 and 2005. Only patients with IDC of no special type, NST (40 in the training set and 331 in the validation set) were included in the study due to the small numbers of patients with other tumour subtypes. Immunohistochemical data were available on all the 40 patients of the training set. While in the validation set, from 331 cases, immunohistochemical data were available on a maximum of 275 patients due to loss of some tissue microarray (TMA) cores during antigen retrieval and staining, a well-recognised phenomenon associated with TMA immunohistochemistry. Clinical history and tumour characteristics (age, tumour type, size, histological grade, lymph node status and Nottingham prognostic index (NPI)) were available. DFS was defined as the interval (months) from primary surgical treatment to the first loco regional or distant recurrence. OS was taken as time (months) from primary surgical treatment to time of death from breast cancer.

\section{Generation of anti-CSMD1 antibody}

Two peptide sequences HFTSDSNHRRKGFN and QRVTE TLAAWSDHR from the N-terminus of human CSMD1 were selected as targets for generating a polyclonal antibody. In vitro synthesis of the peptides and immunisation of chickens with keyhole limpet hemocyanine-conjugated CSMD1 peptides, followed by Ig purification from the egg yolks, were performed by Abcam (Cambridge, UK).

Anti-CSMD1 antibody validation

Initially, the binding specificity of the anti-CSMD1 antibody was tested by an enzyme-linked immunosorbent assay (ELISA) against the BSA-conjugated peptides stated earlier [30]. Antibody specificity was also confirmed on histological tissue sections by pre-absorption with the peptides. The antibody was diluted to its working concentration and incubated with a tenfold excess of relevant peptide overnight at $4{ }^{\circ} \mathrm{C}$ before application to the tissue.

For further validation of the specificity and sensitivity of the antibody and to control the effects of formalin fixation 
and antigen retrieval, CSMD1 was silenced in the MCF10A cell line (ATCC, Manassas, VA, USA) using short hairpin RNA (shRNA). Cells were plated at $1 \times 10^{6}$ cells $/ \mathrm{ml}$ in $6-$ well plates. Next day, they were transfected with $\mathrm{pRS}$ vector $\left(\mathrm{HuSH}^{\mathrm{TM}}\right.$, ORIGENE, Rockville, MD, USA) containing shRNA sequences against CSMD1 mRNA using Lipofectamine $^{\text {TM }} 2000$ (Invitrogen, Paisley, UK). Transfected cells were selected by incubation in DMEM-F12 media (Invitrogen) that contains $0.5 \mu \mathrm{g}$ puromycin (SIGMA-ALDRICH, Dorset, UK) for 2 weeks. To obtain a homogenous cell population and to avoid copy number variation, single cells were plated into the wells of a 96-well plate and grown up to give single-cell colonies. All colonies were then screened by qRT-PCR. Colonies that showed the highest level of knockdown were formalin fixed and paraffin embedded and used as a control for antibody optimisation. To optimise the CSMD1 staining protocol, full sections of paraffin-embedded tissue from both normal and tumour samples were stained using a range of primary antibody dilutions, incubation times and antigen retrieval methods.

Tissue microarrays construction and immunohistochemistry

CUB and SUSHI multiple domains-1 (CSMD1) was stained on full sections of patient's samples in the training set. Whereas in the validation set, tissue microarrays (TMAs) were constructed from the breast cancer patients using $0.6 \mathrm{~mm}$ cores selected from the most representative tumour area. Immunohistochemical analysis of CSMD1 expression was performed with the chicken anti-CSMD1 antibody at a 1:3,000 dilution. TMA sections measuring $4 \mu \mathrm{m}$ were deparaffinised in graded alcohols. Endogenous peroxide activity was inhibited by immersing the sections in $0.5 \%$ $\mathrm{H}_{2} \mathrm{O}_{2}$. Antigen retrieval was carried out by pressure-cooking slides for $90 \mathrm{~s}$ in $1 \%$ Antigen Unmasking Solution (Vector Laboratories Ltd, Peterborough, UK). A casein incubation (1/2 dilution) was also carried out to reduce non-specific staining (Vector Laboratories). Sections were incubated with the antibody for $2 \mathrm{~h}$ at room temperature. Bound antibody was detected with EnVision ${ }^{\mathrm{TM}}$ polymer (DAKO, Ely, Cambridgeshire, UK), using diaminobenzine as the substrate (Sigma, Poole, Dorset, UK). Sections were counterstained with Mayer's haematoxylin (VWR International Ltd, Poole, UK). Negative controls, in which the pre-immune serum was applied, and positive controls of normal breast tissue were included in each batch of immunohistochemistry.

Immunohistochemical evaluation

Cytoplasmic and membranous staining was scored as a percentage of the positive cells in relation to the total number of tumour cells present. Expression status was dichotomised and the cut-off point that showed the biggest difference between patients groups with regard to survival was chosen. Cases were scored blindly and independently by two authors (M. K. and A. M. S., a specialist consultant breast histopathologist). Discordant results were re-evaluated jointly to reach consensus.

\section{Statistical analysis}

Using SPSS version 15.0, Pearson correlation was used to correlate CSMD1 staining as a percentage of the positive cells versus tumour size, age of patient, number of positive nodes, OS time and DFS time as continuous variables. The chi-square test/Fisher exact test or Mann-Whitney $U$-test (non-parametric) was used for comparisons between groups/categorical data as needed. Survival curves were calculated using the Kaplan-Meier method, and differences between groups were analysed by the log-rank test and confirmed by applying Cox regression model. The optimal cut-off for immunohistochemical expression was evaluated by testing different cut-off values versus OS using KaplanMeier curves. For systematic modelling, a stepwise multivariable Cox regression model was used, testing the independent prognostic relevance of CSMD1 immunoreactivity. All statistical tests were two-sided, and a $P \leq 0.05$ value was considered statistically significant.

\section{Results}

Anti-CSMD1 antibody characterisation

Anti-CSMD1 antibody specificity was confirmed by ELISA (supplementary Fig. 1a). The staining pattern of CSMD1 was abolished when the antibody was incubated in the presence of the peptides it was raised against (supplementary Fig. 1b). To test the validity of the antibody to stain paraffin-embedded tissues, the antibody was tested on paraffin-embedded CSMD1 shRNA stable MCF10A cells that showed very weak staining relative to the control shRNA (supplementary Fig. 1c). Anti-CSMD1 antibody was then optimised to stain paraffin-embedded tissues on full sections. Since the CSMD1 staining was homogenous on full sections, we were able to use it to stain TMAs. Optimal results were identified at a 1:3,000 antibody dilution and pressure cooking for $90 \mathrm{~s}$ in unmasking solution for antigen retrieval. The vast majority of normal tissues showed CSMD1 staining providing positive controls.

Patient's characteristics

This study included two groups of patients after excluding non-IDC tumour subtypes, core loss and unscorable cores. 
Table 1 Tumour characteristics

\begin{tabular}{|c|c|}
\hline Variables & Validation set $n=275(\%)$ \\
\hline \multicolumn{2}{|l|}{ Age } \\
\hline Median & 57 \\
\hline Range & $27-92$ \\
\hline \multicolumn{2}{|c|}{ Age distribution } \\
\hline$<50$ & $91(33.1)$ \\
\hline$>50$ & $184(66.9)$ \\
\hline \multicolumn{2}{|c|}{ Tumour size (mm) } \\
\hline Median & 20 \\
\hline Range & $1-100$ \\
\hline \multicolumn{2}{|c|}{ Size distribution (mm) } \\
\hline$<20$ & $160(58.2)$ \\
\hline$>20$ & $108(39.3)$ \\
\hline Missing & $7(2.5)$ \\
\hline \multicolumn{2}{|c|}{ Tumour grade } \\
\hline G1 & $38(13.8)$ \\
\hline G2 & $114(41.5)$ \\
\hline G3 & $123(44.7)$ \\
\hline \multicolumn{2}{|c|}{ Lymph node status } \\
\hline Positive & $144(52.4)$ \\
\hline Negative & $121(44)$ \\
\hline Missing & $10(3.6)$ \\
\hline \multicolumn{2}{|c|}{ Nottingham prognostic index } \\
\hline Good & $60(21.8)$ \\
\hline Moderate & $139(50.5)$ \\
\hline Poor & $64(23.3)$ \\
\hline Missing & $12(4.4)$ \\
\hline
\end{tabular}

The first group had 40 patients and is considered as a training set, while the second group had 275 patients and is considered as a validation set. Detailed patient characteristics of the validation set are summarised in Table 1 .

We dichotomised the percentage of CSMD1 staining. A cut-off point of $30 \%$ showed the most significant difference between the two groups of patients (low and high CSMD1) in overall survival in the validation study and $10 \%$ in the training study. There was not enough follow-up information to meet the statistical analysis requirements in the training set. However, in the validation set, during a median followup period of 107 months (range, 1-152 months), death occurred in 99/275 patients (36\%) and metastasis or/and recurrence occurred in 59/275 patients (21.5\%).

CSMD1 expression in normal and tumour breast tissues

CUB and SUSHI multiple domains-1 (CSMD1) expression pattern was determined in breast epithelium by immunohistochemistry on 10 paraffin embedded normal breast samples. The staining pattern of CSMD1 was luminal, membranous and cytoplasmic. Luminal staining is shown in Fig. 1a. In the tumour samples, CSMD1 expression exhibited both cytoplasmic and cell membrane staining (Fig. 1b, c).

In the training set, low CSMD1 expression was identified in 22/40 (55\%). Low CSMD1 expression is shown in an IDC sample (Fig. 1e). While in the validation set, 79/ 275 (28.7\%) of cases showed low CSMD1 expression. Low and high CSMD1 expression in TMA samples with IDC are shown in Fig. 1f-i. The adjacent normal epithelium and blood vessels, which have been used as internal controls, showed high CSMD1 staining in the majority of cases. Interestingly, high CSMD1 expression is observed in the normal tissues, and the intact ducts in the tumour samples exhibit low CSMD1 expression (Fig. 1d).

As chromosome $8 \mathrm{p}$, and in particular $8 \mathrm{p} 23$, is frequently deleted in tumours, we wanted to check whether the low CSMD1 expression we were observing in our breast cancer samples was caused by the specific loss of CSMD1 and not simply a consequence of larger non-specific deletions in this region. We did this by retesting a subset of our samples with an antibody raised to MCPH1 (encoded by a neighbouring 8p23 gene (http://genome.ucsc.edu/index.html)). In total, 28 samples were stained with both antibodies. Low expression of CSMD1 was detected in $64 \%$ of cases (18/ 28 ), while low expression of MCPH1 was detected in $25 \%$ of cases (7/28). Low expression of both proteins in the same sample was shown in $18 \%$ of cases $(5 / 28)$.

Relation of CSMD1 expression to histological parameters

In each patient sample, the level of CSMD1 expression was correlated with the severity of the disease. Low expression of CSMD1 was significantly associated with higher tumour grade $(P<0.001$ in the training set and $P=0.003$ in the validation set). In the training set, low expression of CSMD1 was identified in $4.45 \%(1 / 22)$ in grade $1,40.9 \%$ $(9 / 22)$ in grade 2 and $54.4 \%(12 / 22)$ in grade 3 . In the validation set, low expression of CSMD1 was identified in $21.5 \%$ (17/79), $25.58 \%$ (21/79) and $51.89 \%$ (41/79) in grade 1,2 , and 3 , respectively. Low or high CSMD1 expressing tumours did not differ significantly for the distribution of other clinicopathological variables (Table 2).

Univariate and multivariate analyses

In the validation set, low expression of CSMD1 was significantly associated with shorter OS, with an estimated median OS of 65 months (95\% CI: 38.8-91.1) compared to 135 months in patients with high CSMD1 expression $(P=0.01$; Fig. 2$)$. There was no significant association between CSMD1 expression and DFS $(P=0.48)$. However, the mean DFS in patients with low CSMD1 was 

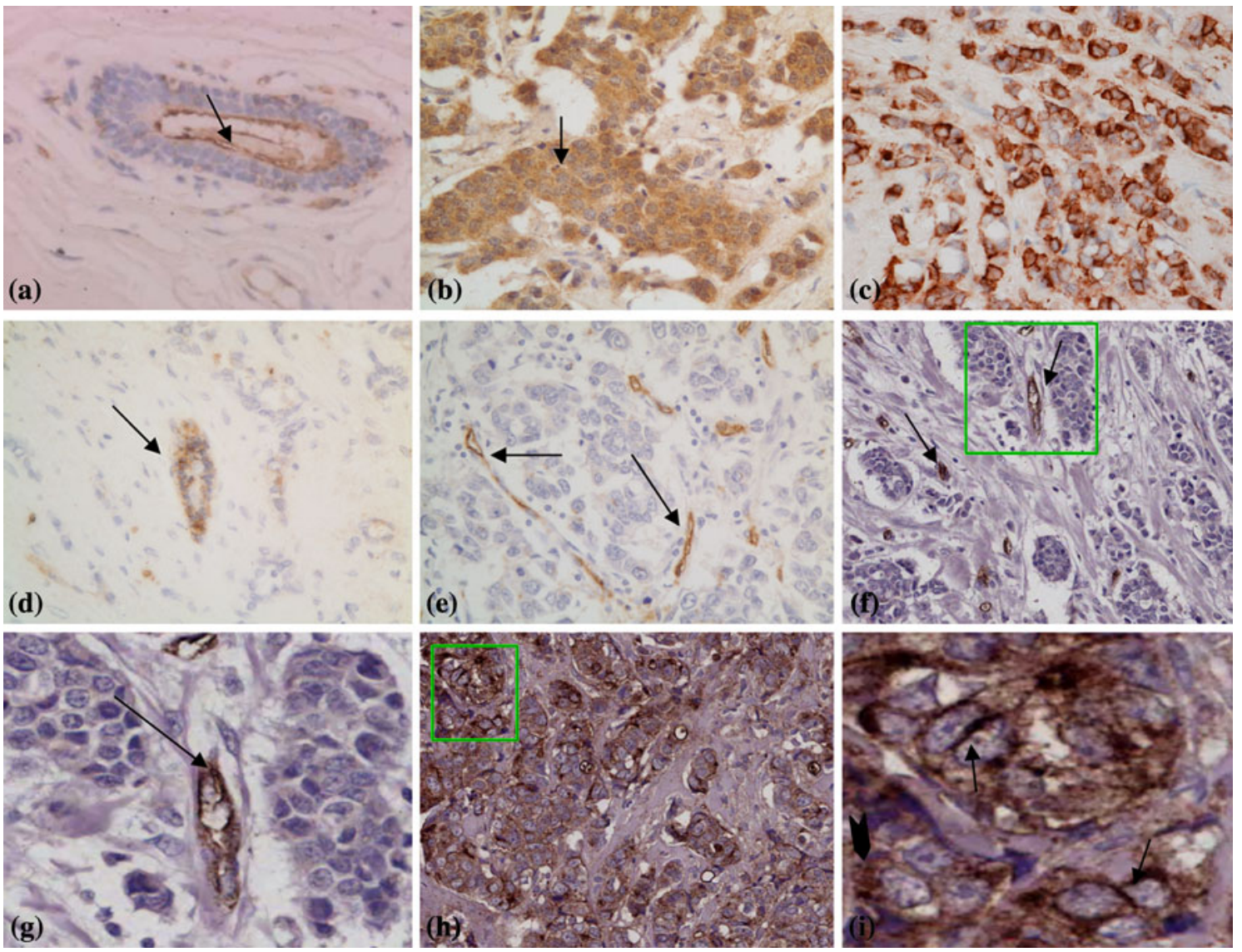

Fig. 1 Immunohistochemical analysis of CSMD1 expression in normal and malignant breast samples. a Normal mammary duct showing luminal cytoplasmic expression of CSMD1 (arrow) $(\times 40)$. b Invasive ductal carcinoma showing cytoplasmic staining (arrow) $(\times 40)$. c Invasive ductal carcinoma showing strong membranous staining $(x 40)$. d Invasive ductal carcinoma, the malignant cells shows no expression, but the normal duct shows cytoplasmic staining (arrow) $(\times 40)$. e Invasive ductal carcinoma with no CSMD1 expression. Blood vessels (internal controls) positive expression

108.7 months (95\%CI: 93.4-123.9) compared to 120 months (95\%CI: 110.9-128.1) of those who highly express CSMD1 (Fig. 3). Univariate analysis showed significant association between CSMD1 expression and OS (HR $=0.607,95 \%$ CI: $0.4-0.91, P=0.018$; Table 3 ) but not with DFS $(\mathrm{HR}=0.81,95 \% \mathrm{CI}$ : $0.46-1.43, P=0.48$; Table 4).

A multivariate Cox regression model was carried out to examine the prognostic impact of CSMD1 expression on OS and DFS in relation to other established clinicopathological and tumour-biological factors such as tumour grade, lymph node status and NPI. CSMD1, together with NPI, appeared to be independent predictors of OS $(\mathrm{HR}=0.6$, 95\%CI: $0.3-0.9, P=0.03$; Table 3). However, unlike tumour grade, CSMD1 was not shown to be an independent predictor of DFS $\quad(\mathrm{HR}=0.84, \quad 95 \% \mathrm{CI}: \quad 0.46-1.5$, $P=0.573$; Table 4). (arrows) $(\times 40)$. f Invasive ductal carcinoma TMA showing low level of CSMD1 expression. Blood vessels showing positive expression (arrows) $(\times 20)$. g Invasive ductal carcinoma TMA (magnification of box in f) showing low level of CSMD1 expression with positive expression in the blood vessels (arrow) $(\times 40)$. h Invasive ductal carcinoma TMA showing high level of CSMD1 expression $(\times 20)$. i Invasive ductal carcinoma TMA (magnification of box in f) showing high level of CSMD1 expression in both cell membranes (arrows) and cytoplasm (arrow head) $(\times 40)$

\section{Discussion}

The short arm of chromosome $8 \mathrm{p}$ is frequently deleted in a wide range of cancers including breast cancer [7-15]. Interestingly, $8 \mathrm{p} 23$ deletions are associated with poor survival in patients with various types of cancers including breast cancer [10,13,14, 20,31-35]. The properties of CSMD1 make it one candidate for the putative $8 \mathrm{p} 23$ tumour suppressor genes [5]. In this study, we have undertaken the first investigation of the role of CSMD1 in breast cancer. We have developed an anti-CSMD1 antibody and investigated its protein distribution in normal and malignant breast tissues.

The specificity of our anti-CSMD1 antibody was confirmed by ELISA and incubation in the presence/absence of the peptides it was raised against. Unfortunately, our antibody is not suitable for western blot analysis. However, 
Table 2 Clinicopathological parameters in relation to CSMD1

\begin{tabular}{|c|c|c|c|}
\hline \multirow[t]{2}{*}{ Variables } & \multicolumn{3}{|c|}{ CSMD1 expression $^{\mathrm{a}}$} \\
\hline & Low $n(\%)$ & High $n(\%)$ & $P$ value \\
\hline \multicolumn{4}{|c|}{ Age distribution } \\
\hline$<50$ & $24(30.37)$ & $67(34.18)$ & \multirow[t]{2}{*}{0.54} \\
\hline$>50$ & $55(69.62)$ & $129(65.81)$ & \\
\hline \multicolumn{4}{|c|}{ Size distribution (mm) } \\
\hline$<20 \mathrm{~mm}$ & $50(65.78)$ & $110(57.29)$ & \multirow[t]{2}{*}{0.20} \\
\hline$>20 \mathrm{~mm}$ & $26(34.21)$ & $82(42.70)$ & \\
\hline \multicolumn{4}{|l|}{ Tumour grade } \\
\hline G1 & $17(21.51)$ & $21(10.7)$ & \multirow[t]{3}{*}{0.003} \\
\hline $\mathrm{G} 2$ & $21(26.58)$ & $93(47.4)$ & \\
\hline G3 & $41(51.89)$ & $82(41.83)$ & \\
\hline \multicolumn{4}{|c|}{ Lymph node status } \\
\hline Positive & $34(45.94)$ & $87(45.54)$ & \multirow[t]{2}{*}{0.95} \\
\hline Negative & $40(54.05)$ & $104(54.45)$ & \\
\hline \multicolumn{4}{|c|}{ Nottingham prognostic index } \\
\hline Good & $21(28.76)$ & $39(20.52)$ & \multirow[t]{3}{*}{0.31} \\
\hline Moderate & $34(46.57)$ & $105(55.26)$ & \\
\hline Poor & $18(24.65)$ & $46(24.21)$ & \\
\hline
\end{tabular}

a $30 \%$ cut-off was used to dichotomise CSMD1 expression into low and high groups

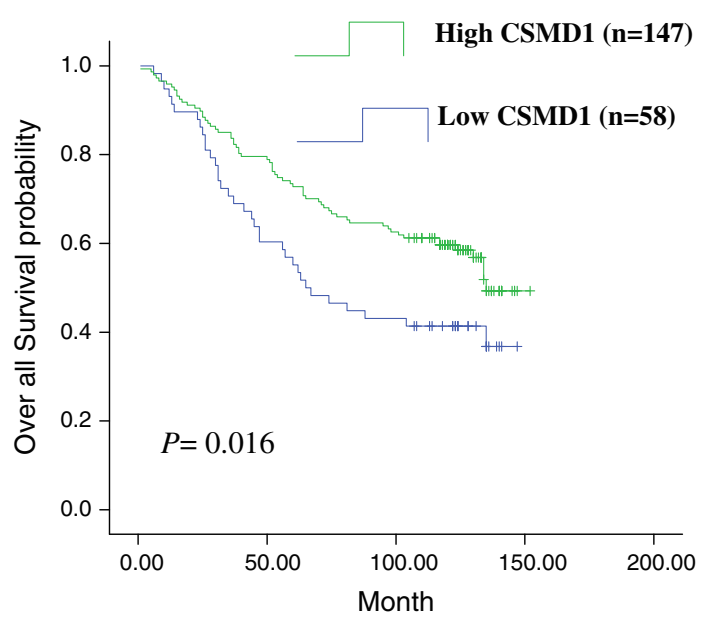

Fig. 2 Comparison of overall survival based on CSMD1 status in the validation set

we have silenced CSMD1 in a breast cell line (MCF10A) using shRNA and shown that these cells show a much weaker staining pattern with the CSMD1 antibody in comparison with control cells.

In normal breast duct epithelial cells, luminal, membranous and cytoplasmic CSMD1 staining was observed. In the tumour cells, membranous and/or cytoplasmic CSMD1 staining was identified. The luminal and cytoplasmic expression may represent expression and/or secretion of protein(s) encoded by alternatively spliced CSMD1 transcripts. The cytoplasmic CSMD1 expression may

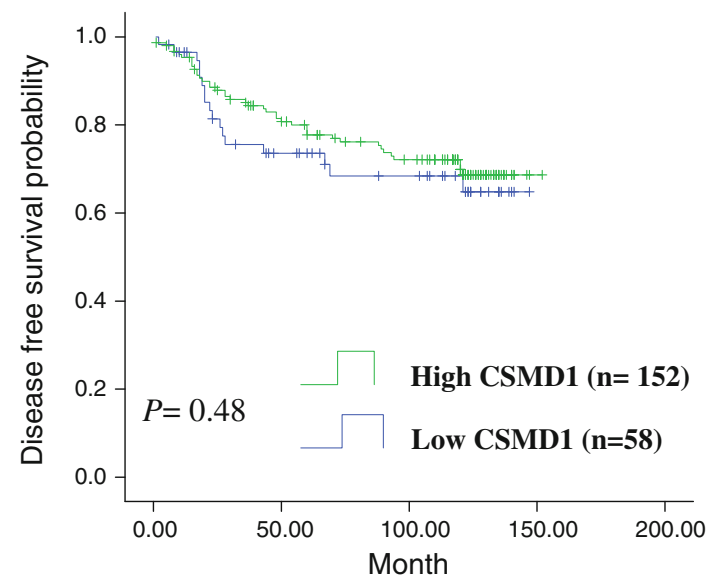

Fig. 3 Comparison of disease-free survival based on CSMD1 status in the validation set

alternatively represent internalisation of the full-length CSMD1 protein. Indeed, the cytoplasmic tail of CSMD1 contains a receptor internalisation motif (FENPMYDT/RN motif) that could potentially mediate clathrin-dependent endocytosis [36]. The CSMD1 membrane-staining pattern is consistent with the presence of a transmembrane domain and is in agreement with its putative role as a receptor or coreceptor playing a role in signal transduction.

In samples that contained both normal and tumour tissues, we observed higher CSMD1 expression in the normal tissues and intact ducts in comparison with the surrounding tumour cells. This raises the question of whether reduction of CSMD1 expression is a consequence or a cause of the disrupted tissue morphology. Studies on the rat ortholog of CSMD1 have shown that CSMD1 expression is low in brain regions exhibiting high levels of cell proliferation and very high in regions of neuronal differentiation, suggesting that CSMD1, like neuropilin and other neuronal growth cone proteins, may be involved in mechanisms of signal transduction, substrate adhesion and/or motility that help guide axons towards their synaptic targets during development [22].

Initially, we recorded low CSMD1 expression in 55\% $(22 / 40)$ of the training set. Our larger validation set also showed a reduction in CSMD1 expression, however: the percentage loss dropped to $28.7 \%$ (79/275). Our results agree with other studies that report reduced CSMD1 mRNA expression in prostate cancer, hepatocellular carcinoma, HNSCCs and lung SCC [17, 19, 20].

In this study, we evaluated the protein expression of CSMD1 in breast cancer but have not experimentally determined the way in which the CSMDI gene may be lost or inactivated. Epigenetic modification of the genomic DNA preceding the first exon of CSMD1 has been shown to be associated with its silencing in squamous cancers [37], and aberrant splicing and point mutations have also been shown 
Table 3 Univariate and multivariate analyses of overall survival in the validation set

\begin{tabular}{|c|c|c|c|c|}
\hline Variable & Univariate ( $P$ value $)$ & HR $(95 \% \mathrm{CI})$ & Multivariate ( $P$ value) & HR $(95 \% \mathrm{CI})$ \\
\hline \multicolumn{5}{|l|}{ Grade } \\
\hline G1 vs. G2 vs. G3 & 0.000 & $1.78(1.28-2.45)$ & 0.34 & $1.2(0.79-1.97)$ \\
\hline \multicolumn{5}{|l|}{ Lymph node status } \\
\hline Negative vs. positive & 0.009 & $1.79(1.16-2.76)$ & 0.947 & $0.98(0.53-1.8)$ \\
\hline \multicolumn{5}{|l|}{ NPI } \\
\hline Good vs. moderate vs. poor & 0.000 & $2.09(1.53-2.87)$ & 0.015 & $1.96(1.14-3.36)$ \\
\hline \multicolumn{5}{|l|}{ CSMD1 } \\
\hline High vs. low ${ }^{\mathrm{a}}$ & 0.018 & $0.607(0.4-0.91)$ & 0.03 & $0.6(0.3-0.9)$ \\
\hline
\end{tabular}

Multivariate analysis: $n=195$; deceased $=90$, censored $=105$, missing $=80$

a $30 \%$ cut-off was used to dichotomise CSMD1 expression into low and high groups

Table 4 Univariate and multivariate analyses of DFS in the validation set

\begin{tabular}{|c|c|c|c|c|}
\hline Variable & Univariate ( $P$ value $)$ & HR $(95 \% \mathrm{CI})$ & Multivariate ( $P$ value $)$ & HR $(95 \% \mathrm{CI})$ \\
\hline \multicolumn{5}{|l|}{ Grade } \\
\hline G1 vs. G2 vs. G3 & 0.000 & $3.9(2.27-6.7)$ & 0.003 & $2.72(1.4-5.26)$ \\
\hline \multicolumn{5}{|l|}{ Lymph node status } \\
\hline Negative vs. positive & 0.000 & $3.8(1.99-7.46)$ & 0.057 & $2.26(0.97-5.25)$ \\
\hline \multicolumn{5}{|l|}{ NPI } \\
\hline Good vs. moderate vs. poor & 0.000 & $3.41(2.21-5.26)$ & 0.167 & $1.6(0.82-3.11)$ \\
\hline \multicolumn{5}{|l|}{ CSMD1 } \\
\hline High vs. low ${ }^{\mathrm{a}}$ & 0.48 & $0.81(0.46-1.43)$ & 0.573 & $0.84(0.46-1.5)$ \\
\hline
\end{tabular}

Multivariate analysis: total $n=200$; deceased $=57$, censored $=143$, missing $=75$

a $30 \%$ cut-off was used to dichotomise CSMD1 expression into low and high groups

to lead to loss of CSMD1 in a variety of cancers $[16,18]$. However, deletion of the genomic region encompassing CSMD1 is probably the most common mechanism. Chromosome 8p12-ptel deletions are commonly found in multiple cancers and intragenic homozygous deletions in CSMD1 have been reported in oral SCC [11]. Similarly, array-based CGH studies have detected loss of CSMDI in HNSCCs, lung SCC and breast cancers, cutaneous SCC and basal cell carcinomas. While we believe deletion of CSMD1 is the most common mechanism of inactivation, we wanted to rule out that we were detecting random deletion of $8 \mathrm{p} 23$. To prove this, we stained a cohort of our IDCs with an antibody for a neighbouring protein, MCPH1. A far higher proportion of samples showed low CSMD1 expression compared to MCPH1 and only $18 \%$ of these cases showed reduced expression of both proteins, indicating that reduction of CSMD1 expression is specific.

Our results revealed that low CSMD1 expression was significantly associated with high tumour grade in both our sample sets. In the validation set, reduction of CSMD1 expression was significantly associated with shorter OS. This agrees with other studies that have reported an association between loss of the 8pter-p23 region or CSMD1 and poor survival and high stage disease in prostate, bladder and head and neck cancer patients. This suggests that CSMD1 may be a marker of advanced stage disease and disease recurrence [20,32, 33, 35].

Multivariate Cox regression model showed that CSMD1 expression and NPI both appeared to be independent predictors of OS. This result agrees with a study in supraglottic SCC where allelic loss at 8p23 appears to be a statistically significant independent predictor of poor prognosis [13]. However, unlike tumour grade, CSMD1 expression was not shown to be an independent predictor of DFS. This result is in contrast to an earlier study of prostate cancer that showed that loss at $8 \mathrm{p} 23.2$ is a predictive indicator of postoperative recurrence independent of stage and grade [19]. Further large confirmatory studies are warranted to determine the potential use of CSMD1 expression assessment of clinical practice in breast and other cancer types.

In summary, the predominant staining pattern of CSMD1 was identified on the luminal epithelial cells, which supports the idea that CSMD1 may be a receptor or co-receptor involved in the process of signal transduction. Moreover, the high CSMD1 expression levels observed in the well-differentiated tumours compared to the poorly 
differentiated areas, suggests that CSMD1 may play a role in cell morphogenesis and/or cell differentiation and duct formation. Reduction of CSMD1 expression was recorded in almost a third of breast carcinoma cases and significantly associated with high tumour grade and decreased OS. Together with the multivariate analysis, which identified CSMD1 as an independent prognostic factor for OS, the present results provide clinical evidence to support the idea that CSMD1 is a tumour suppressor gene, which may prove to be a new prognostic biomarker.

Acknowledgments This study was supported by grants from Yorkshire Cancer Research and Breast Cancer Campaign (SMB \& VS), the Association for International Cancer Research (LZ \& NT) and the Egyptian government (MK). CT is a Royal Society University Research Fellow.

\section{References}

1. Bray F, Sankila R, Ferlay J et al (2002) Estimates of cancer incidence, mortality in Europe in 1995. Eur J Cancer 38(1):99166

2. Parkin DM, Bray F, Ferlay J et al (2001) Estimating the world cancer burden: globocan 2000. Int J Cancer 94(2):153-156

3. Fabre-Lafay S, Monville F, Garrido-Urbani S et al (2007) Nectin4 is a new histological and serological tumor associated marker for breast cancer. BMC Cancer 7(1):73

4. Alford D, Taylor-papadimitrion J (1996) Cell adhesion molecules in the normal and cancerous mammary gland. J Mammary Gland Biol Neoplasia 1(2):207-218

5. Sun PC, Uppaluri R, Schmidt AP et al (2001) Transcript map of the 8p23 putative tumor suppressor region. Genomics 75(13): $17-25$

6. Kirkitadze MarinaD, NB P (2001) Structure and flexibility of the multiple domain proteins that regulate complement activation. Immunol Rev 180(1):146-161

7. Sigbjornsdottir BI, Ragnarsson G, Agnarsson BA et al (2000) Chromosome $8 \mathrm{p}$ alterations in sporadic and BRCA2 999del5 linked breast cancer. J Med Genet 37(5):342-347

8. Yokota T, Yashimoto M, Akiyama F et al (1999) Localization of a tumor suppressor gene associated with the progression of human breast carcinoma within a $1-\mathrm{cm}$ interval of $8 \mathrm{p} 22-\mathrm{p} 23.1$. Cancer 85(2):447-452

9. Emi M, Fujiwara Y, Nakajima T et al (1992) Frequent loss of heterozygosity for loci on chromosome $8 \mathrm{p}$ in hepatocellular carcinoma, colorectal cancer, and lung cancer. Cancer Res 52(19):5368-5372

10. Wu CL, Roz L, Sloan P et al (1997) Deletion mapping defines three discrete areas of allelic imbalance on chromosome arm $8 \mathrm{p}$ in oral and oropharyngeal squamous cell carcinomas. Genes. Chromosom Cancer 20(4):347-353

11. Toomes C, Jackson A, Maguire K et al (2003) The presence of multiple regions of homozygous deletion at the CSMD1 locus in oral squamous cell carcinoma question the role of CSMD1 in head and neck carcinogenesis. Genes. Chromosom Cancer 37(2):132-140

12. Sunwoo JB, Holt MS, Radford DM et al (1996) Evidence for multiple tumor suppressor genes on chromosome arm 8p in supraglottic laryngeal cancer. Genes Chromosomes Cancer 16(3):164-169
13. Scholnick SB, Haughey BH, Sunwoo JB et al (1996) Chromosome 8 allelic loss and the outcome of patients with squamous cell carcinoma of the supraglottic larynx. J Natl Cancer Inst 88(22):1676-1682

14. Fujiwara Y, Emi M, Ohata $\mathrm{H}$ et al (1993) Evidence for the presence of two tumor Suppressor genes on chromosome $8 \mathrm{p}$ for colorectal carcinoma. Cancer Res 53(5):1172-1174

15. Ishwad CS, Shuster M, Buckmuhl U et al (1999) Frequent allelic loss and homozygous deletion in chromosome band $8 \mathrm{p} 23$ in oral cancer. Int J Cancer 80(1):25-31

16. Ma C, Quesnelle KM, Sparano A et al (2009) Characterization CSMD1 in a large set of primary lung, head and neck, breast and skin cancer tissues. Cancer Biol Ther 8(10):29-38

17. Midorikawa Y, Yamamato S, Tsuji S et al (2008) Allelic imbalances and homozygous deletion on 8p23.2 for stepwise progression of hepatocarcinogenesis. Hepatology 49(2):513-522

18. Farrell LC, Crimm H, Meeh P et al (2008) Somatic mutations to CSMD1 in colorectal adenocarcinomas. Cancer Biol Ther 7(4): 609-613

19. Henshall SM, Afar DEH, Hiller J et al (2003) Survival analysis of genome-wide gene expression profiles of prostate cancers identifies new prognostic targets of disease relapse. Cancer Res 63(14): 4196-4203

20. Paris PL, Andaya A, Fridlyand J et al (2004) Whole genome scanning identifies genotypes associated with recurrence and metastasis in prostate tumors. Hum Mol Genet 13(13):1303-1313

21. Nishioka M, Kohno T, Takahashi M et al (2000) Identification of a 428-kb homozygously deleted region disrupting the SEZ6L gene at 22q12.1 in a lung cancer cell line. Oncogene 19(54): $6251-6260$

22. Kraus DM, Elliott GS, Chute H et al (2006) CSMD1 is a novel multiple domain complement-regulatory protein highly expressed in the central nervous system and epithelial tissues. J Immunol 176(7):4419-4430

23. Mollenhauer J, Wiemann S, Scheurlen W et al (1997) DMBT1, a new member of the SRCR superfamily, on chromosome 10q25. $3-26.1$ is deleted in malignant brain tumours. Nat Genet 17(1): 32-39

24. Wu W, Kemp BL, Proctor ML et al (1999) Expression of DMBT1, a candidate tumor suppressor gene, is frequently lost in lung cancer. Cancer Res 59(8):1846-1851

25. Mollenhauer J, Helmke B, Medina D et al (2004) Carcinogen inducibility in vivo and down-regulation of DMBT1 during breast carcinogenesis. Genes Chromosom Cancer 39(3):185-194

26. Bork P, Beckmann G (1993) The CUB domain: a widespread module in developmentally regulated proteins. J Mol Biol 231(2):539-545

27. Topfer-Petersen E, Romero A, Varela PF et al (1998) Spermadhesins: a new protein family. Facts, hypotheses and perspectives. Andrologia 30(4-5):217-224

28. Lu S-L, Kawabata M, Imamura T et al (1998) HNPCC associated with germline mutation in the TGF-[beta] type II receptor gene. Nat Genet 19(1):17-18

29. Su GH, Bansal R, Murphy KM et al (2001) ACVR1B (ALK4, activin receptor type $1 \mathrm{~B}$ ) gene mutations in pancreatic carcinoma. Proc Natl Acad Sci U S A 98(6):3254-3257

30. Williams LM, Drew JE, Bunnett NW et al (2001) Characterization of an antibody to the human melatonin mt1 receptor. J Neuroendocrinol 13(1):94-101

31. Muscheck M, Sukosd F, Pesti T et al (2000) High density deletion mapping of bladder cancer localizes the putative tumor suppressor gene between loci D8S504 and D8S264 at chromosome 8p23.3. Lab Invest 80(7):1089-1093

32. Washburn JG, Wojno KJ, Dey J et al (2000) 8pter-p23 deletion is associated with racial differences in prostate cancer outcome. Clin Cancer Res 6(12):4647-4652 
33. Bockmühl U, Ishwad CS, Ferrell RE et al (2001) Association of 8p23 deletions with poor survival in head and neck cancer. Otolaryngol Head Neck Surg 124(4):451-455

34. Weber-Mangal S, Sinn H, Popp S et al (2003) Breast cancer in young women ( $<$ or $=35$ years): genomic aberrations detected by comparative genomic hybridization. Int J Cancer 107(4):583-592

35. Wright K, Wilson PJ, Kerr J et al (1998) Frequent loss of heterozygosity and three critical regions on the short arm of chromosome 8 in ovarian adenocarcinomas. Oncogene 17(9): $1185-1188$

36. Paccaud JP, Reith W, Johansson B et al (1993) Clathrin-coated pit-mediated receptor internalization. Role of internalization signals and receptor mobility. J Biol Chem 268(31):23191-23196

37. Richter T, Tong B, Scholnick S (2005) Epigenetic inactivation and aberrant transcription of CSMD1 in squamous cell carcinoma cell lines. Cancer Cell Int 5(1):29 